\title{
Das disruptive Potential der Blockchain-Technologie
}

\author{
Markus Hablizel
}

\begin{abstract}
Das Geschäftsmodell eines Versicherers beruht auf einem zentralen Element: Vertrauen. Vertrauen des Kunden auf Hilfe im Schadensfall; Vertrauen auf die richtige Beratung durch den Vermittler; Vertrauen auf den Versicherer, dass er die ihm übertragenen Risiken angemessen managt; Vertrauen der Investoren und Aktionäre, dass das bereit gestellte Kapital gewinnbringend eingesetzt wird; und zuletzt auch, Vertrauen der

Gesellschaft darauf, dass existenzbedrohende Risiken ihrer Bürger abgesichert werden können.

Zusammengefasst: Ein Versicherer ist der Inbegriff eines Trustbrokers. Dieses auf Vertrauen basierende Geschäftsmodell wird durch die Blockchain erschüttert.

In diesem Artikel werden die Auswirkungen der Blockchain-Technologie mit einem besonderen Fokus auf

die Versicherungsbranche diskutiert. Da die Technologie auf Komponenten aus der Mathematik und Informatik basiert, wird ein gewisses Interesse der Leserschaft hieran angenommen. Der Artikel verzichtet

bewusst auf manche technischen Details, sondern soll vielmehr das disruptive Potential aus der geschäftlichen Perspektive aufzeigen.
\end{abstract}

1

Einordnung

Die Blockchain-Technologie ist zweifelsohne eine der bedeutendsten Erfindungen des gegenwärtigen Jahrzehnts. Dies zeigt sich zum Einen an den unzähligen Veröffentlichungen $^{1}$ hierzu, zum Anderen aber auch quantitativ gemessen an den hohen Beträgen, die Risikokapitalgeber bereit waren, in die Technologie zu investieren, wie Abbildung 1 illustriert. Dies spiegelt die Erwartungen an die zukünftigen Möglichkeiten und Potentiale der Technologie wider.

Vor Kurzem hat ebenso eine der einflussreichsten Venture Capital-Firmen, Andreessen Horowitz, verkündet, ${ }^{2}$ über einen Fonds \$300 Millionen in Krypto-Startups investieren zu wollen. Auch im bekannten Hype Cycle 3 der Firma Gartner wird die Blockchain-Technologie als einer von drei technologischen Megatrends mit einer prognosti-

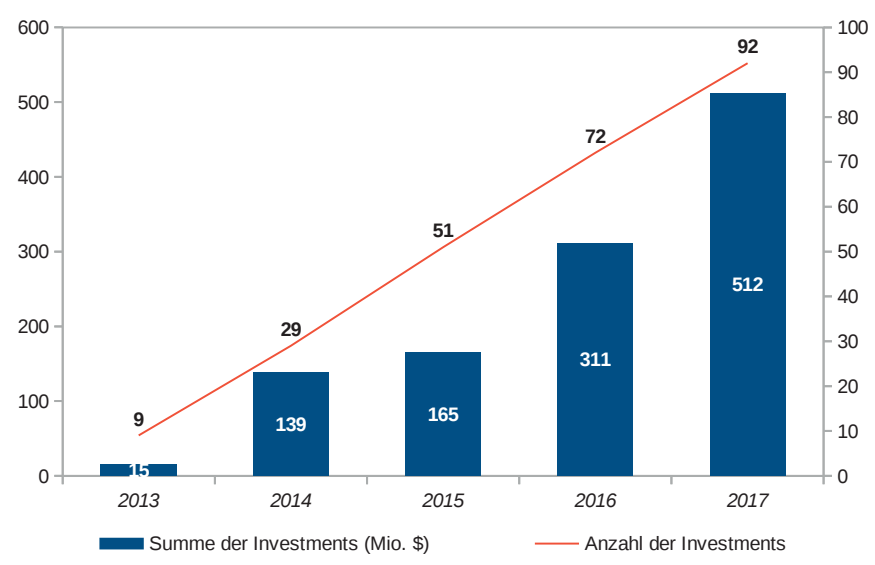

Abbildung 1. Globale Blockchain-Investments 2013-2017 (Datengrundlage: KPMG/Pitchbook) zierten Zeitspanne von 5-10 Jahren bis zur breiten Etablierung und produktiven Nutzung aufgeführt.

Einer der Gründe für dieses in den letzten Jahren stark angestiegene Interesse an der Technologie liegt in der Fülle von Chancen, die die Blockchain als technologische Infrastruktur für einzelne Branchen, aber auch branchenübergreifend verspricht. Wie meist bei Innovationen durch eine neue Technologie wird auch hier erst in der Rückschau das gesamte Ausmaß der Veränderung ersichtlich werden. Der oft zu hörende Vergleich, dass die Blockchain derzeit einen ähnlichen Reifegrad wie das Internet Mitte der 1990er-Jahre besitzt, erscheint mehr als zutreffend. Daher beschäftigen sich nicht nur Unternehmen aus dem IT-Umfeld damit, sondern auch Unternehmen und Branchen, bei denen die Verbindung zur Technologie nicht direkt offensichtlich ist.

2

Was ist die Blockchain-Technologie?

Die erste und bis heute prominenteste Anwendung der Blockchain-Technologie - die Kryptowährung Bitcoin - wurde von einer unbekannten Person (oder Gruppierung), die sich das Pseudonym Satoshi Nakamoto gab, in ihrem bahnbrechenden Artikel [11] aus dem Jahr 2008 beschrieben. Er verwendet hierin verschiedene, bereits seit längerer Zeit existierende Konzepte und Verfahren aus der Informatik und Mathematik, insbesondere Kryptografie, Hashfunktionen, Merkle-Trees sowie Wahrscheinlichkeitsrechnung. Auf den lediglich neun Seiten des Artikels beschreibt der Autor ein System, das eine dezentrale digitale Währung ermöglicht, die ein Double-Spending 4 verhindert. Dies war für den Fall eines dezentralen Systems, das also ohne einen zentralen Intermediär auskommt, das erste seiner Art. Alle früheren Ansätze scheiterten an diesem Problem des Double- 
Spendings. Eine lesenswerte Übersicht über die Anfänge und Herausforderungen digitaler Währungen findet sich im Buch von Narayanan et al. [12].

Satoshi Nakamoto nutzt für Bitcoin eine lineare Verkettung von Blöcken (daher der Name Blockchain), in welchen die einzelnen Transaktionen ${ }^{5}$ gespeichert werden. Die Verkettung erfolgt dabei über die fortlaufende Integration des Hashwerts des vorhergehenden Blocks, vgl. Abbildung 2.

Im einfachsten Fall stellt die Blockchain eine Möglichkeit bereit, ein verteiltes Kontenbuch ${ }^{6}$, das durch mehrere Nutzer gepflegt werden kann, zu führen. In einem solchen Kontenbuch werden Transaktionen, das heißt Veränderungen von dessen Einträgen, dezentral verzeichnet. Als Beispiel kann hier der Wechsel des Eigentumsnachweises an einem Wertgegenstand, wie Geldmittel, angeführt werden. Im Grunde ist dieses Kontenbuch dann nichts anderes als eine verteilte Datenbank, in der mehrere Nutzer Lese- und Schreibrechte besitzen. Die durchgeführten Änderungen und Ergänzungen in dieser Datenbank werden dabei über kryptografische Verfahren abgesichert, so dass sie für die anderen Nutzer transparent 7 nachvollziehbar und unveränderlich sind. Darüber hinaus ist eine vollständige Version des Kontenbuchs auf allen teilnehmenden Rechnern gespeichert und jegliche Veränderung zieht eine entsprechende Synchronisation aller dieser Kopien nach sich. Da diese folglich nicht voneinander abweichen, existiert zu jedem Zeitpunkt immer eine korrekte Version des Kontenbuchs (Single Source of Truth). Um Missbrauch zu verhindern, bedürfen die Änderungen, bevor sie in das Kontenbuch aufgenommen werden, einer Art Genehmigung (Konsens) durch die Teilnehmer, die je nach Anwendungsfall unterschiedlich ausgestaltet sein kann. Die letztgenannten Eigenschaften sind insbesondere dann notwendig, wenn kein vollständiges Vertrauen bzw. ein gewisses Misstrauen unter den Teilnehmern vorhanden ist.

Ein solches Szenario kommt sehr viel häufiger vor, als vielleicht zunächst vermutet: Dies wird deutlich, wenn man sich bewusst macht, dass der Austausch von Information eine digitale Transaktion ist, die über ein verteiltes Kontenbuch durchführbar ist. Natürlich kann dies auch genauso gut über eine zentrale Datenbank erfolgen, jedoch überwiegen in vielen Anwendungsszenarien die Vorteile der dezentralen Variante, insbesondere, wenn mehrere Parteien involviert sind, die sich nicht vollständig vertrauen. Die Blockchain wird aus diesem Grund oft auch als Trust Infrastructure bezeichnet.

\section{Versuch einer Definition}

Auf Grund des jungen Alters der Blockchain-Technologie hat sich bislang keine allgemein akzeptierte Sicht auf diese durchgesetzt. Es finden sich unterschiedliche Ausprägungen, die sich jedoch in den allermeisten Fällen derselben zwei Kernkomponenten bedienen: Der Kryptografie und einer verteilten Datenbank.

Im Sinne einer möglichst umfassenden Definition soll daher unter der Blockchain eine verteilte Datenbank in einem dezentralen Netzwerk, deren Einträge über kryptografische Verfahren unveränderlich gespeichert werden, verstanden werden. Eine plakative Veranschaulichung des Unterschieds zwischen zentralen, dezentralen und verteilten Netzwer$\mathrm{ken}^{8}$ beinhaltet Abbildung 3 .

Diese Definition für Blockchain mag nicht jeden Puristen zufrieden stellen, da auch andere Entwicklungen, wie IOTA $^{9}$ - ohne lineare Verkettung der Blöcke, sondern als gerichteter azyklischer Graph - oder die Corda-Plattform der Firma $\mathrm{R}_{3}{ }^{1 \mathrm{O}}$, darunter fallen. Vor dem praktischen Fokus des vorliegenden Artikels ist diese breite Definition aber die Geeignetste, um die Auswirkungen aus der Unternehmensperspektive zu diskutieren.

Es wird explizit kein konkreter Konsens-Mechanismus in die obige Definition mit aufgenommen. Ein Konsens-Mechanismus, welcher über die Validität von neuen Transaktionen (Datenbankeinträgen) entscheidet, sollte vielmehr je nach konkretem Anwendungsfall passend gewählt werden. Diese Wahl hat weitreichende Implikationen, insbesondere auf die Performanz im Netzwerk. Für eine Darstellung verschiedener Konsens-Mechanismen, siehe Abbildung 4.

Die theoretische Grundlage moderner Konsens-Mechanismen wurde mit Hilfe der Kryptografie, insbesondere di-

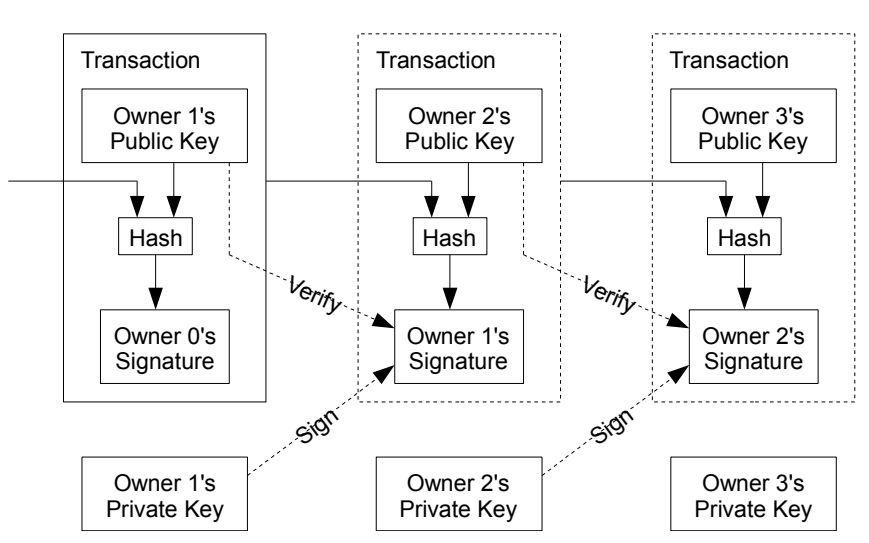

Abbildung 2. Verkettung der Transaktionsblöcke in der BitcoinBlockchain (Quelle: Satoshi Nakamoto [11])
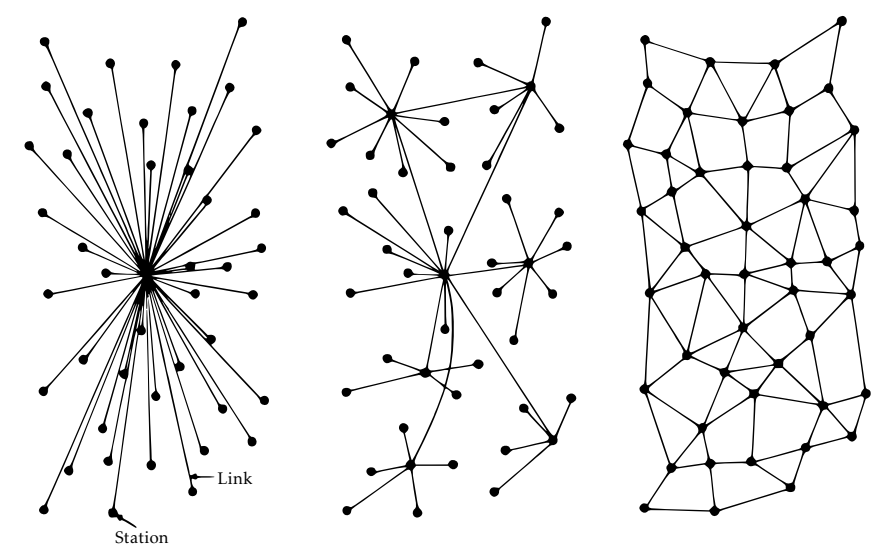

Abbildung 3. Zentrale, dezentrale und verteilte Netzwerke nach Paul Baran [3] (Quelle: openclipart.org/Public domain) 

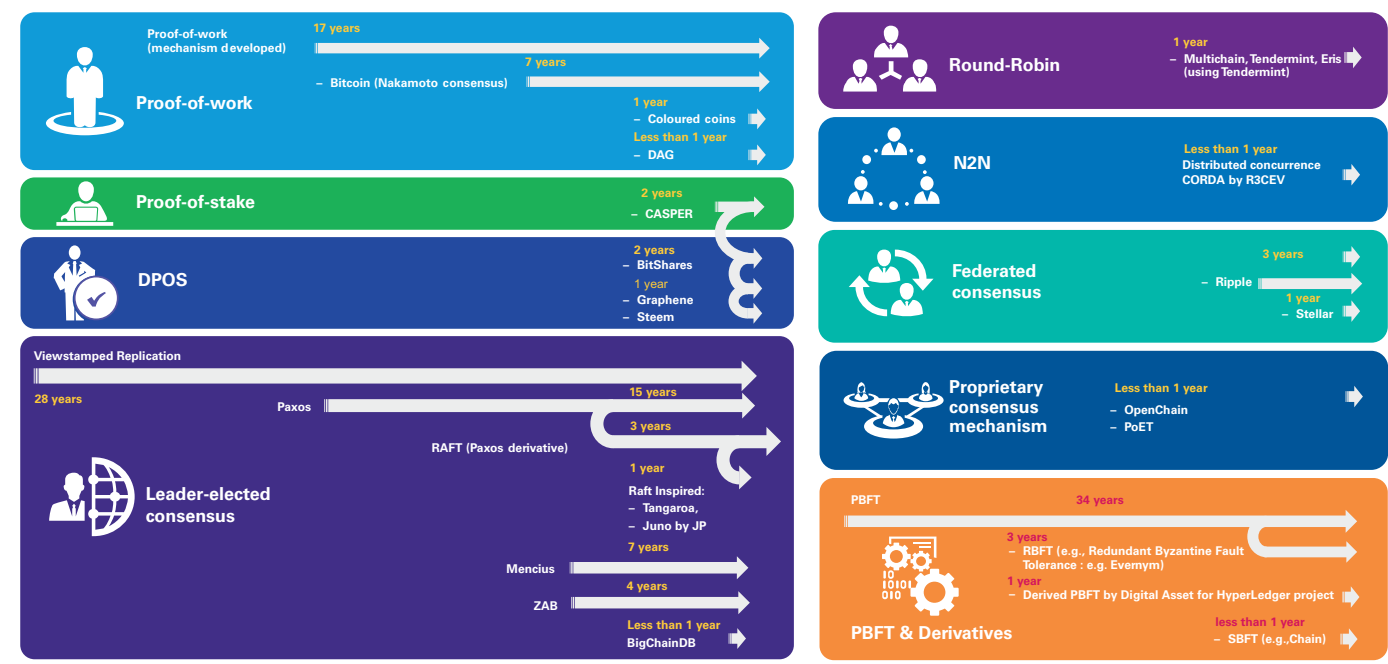

Abbildung 4. Unterschiedliche Konsens-Mechanismen und ihre historische Entwicklung (Quelle: KPMG [8])

gitalen Signaturen, gelegt: In der Veröffentlichung von Paul Baran [3] werden verteilte Netzwerke und deren Ausfallsicherheit diskutiert. Leslie Lamport, Robert Shostak und Marshall Pease [9] führen das Problem der byzantinischen Generäle ${ }^{11}$ ein und liefern wichtige Beiträge zur Verlässlichkeit in verteilten Systemen. Sie geben auch eine Lösung ihres Problems an, wenn sich höchstens ein Drittel der Teilnehmer unehrlich verhalten würde. Die bis dato vorliegenden Lösungen hatten jedoch ein Performanz-Problem, was sie in der Praxis nicht ohne Weiteres einsetzbar machte. Dies wurde erst mit der Einführung des Practical Byzantine-FaultTolerance-Algorithmus (PBFT) durch Miguel Castro und Barbara Liskov möglich. In der Einleitung ihres Artikels [4] aus dem Jahr 1999 schreiben sie mit großem Weitblick:

We believe that Byzantine-fault-tolerant algorithms will be increasingly important in the future because malicious attacks and software errors are increasingly common and can cause faulty nodes to exhibit arbitrary behavior. Whereas previous algorithms assumed a synchronous system or were too slow to be used in practice, the algorithm described in this paper is practical: it works in asynchronous environments like the Internet and incorporates several important optimizations that improve the response time of previous algorithms by more than an order of magnitude.

Mit dem PBFT-Algorithmus lassen sich eine große Anzahl von Peer-to-Peer-Nachrichten mit nur geringer Verzögerungszeit verarbeiten. Dadurch werden verteilte Netzwerke belastbarer gegenüber boshaftem oder falschem Verhalten weniger Teilnehmer.

Eine der ersten Implementierungen des PBFT-Algorithmus ist der Proof-of-Work, der als Konsens-Mechanismus in der Bitcoin-Blockchain von Satoshi Nakamoto verwendet wurde. Kritikpunkte, die gegen die Kryptowährung Bitcoin angeführt werden - wie beispielsweise der hohe Energieverbrauch $^{12}$ oder lange Transaktionszeiten -, sind direk- te Konsequenzen aus der Verwendung des Proof-of-Work. Durch die Auswahl eines anderen Konsens-Mechanismus können diese Probleme umgangen werden, obgleich man dabei dann oft auf die vollständige Dezentralität oder Egalität der Teilnehmer verzichten muss. Dies ist aber im Unternehmenskontext meistens ohne Weiteres hinnehmbar. Eine schematische Darstellung der unterschiedlichen Ausgestaltungsmöglichkeiten einer Blockchain-Architektur ist in Abbildung 5 aufgeführt.

\section{Auswirkungen der Blockchain-Technologie}

Die Blockchain ist eine Technologie, die große Implikationen auf digitale Transaktionen hat. Eine eingeschränkte Betrachtung ihrer Auswirkungen auf Kryptowährungen bzw. Zahlungsvorgänge allein, unterschätzt das Potential gewaltig. Die wichtigste digitale Transaktion heutzutage ist der allgegenwärtige Austausch von Information - und hier setzt Blockchain an. Die Technologie ermöglicht es, Informationen zwischen mehreren Parteien vertrauenswürdig sowie verlässlich auszutauschen und dabei einen Konsens über den gültigen Stand der Datenbank, die verteilt bei den Teilnehmern liegt, herzustellen, ohne - und das ist die eigentliche Innovation! - auf die Hilfe eines Intermediärs (im Englischen vollkommen zu Recht als Trusted Third Party bezeichnet) angewiesen zu sein.

Die Rolle eines Intermediärs ist es üblicherweise, Vertrauen in digitalen Transaktionen und Informationsflüssen zwischen mehreren, untereinander unbekannten Parteien herzustellen. Trusted Third Parties kommen in überaus vielen Bereichen vor; die Gängigsten sollen nachstehend exemplarisch aufgeführt werden:

- Finanzdienstleistungen: Banken, Versicherungsgesellschaften, Makler/Vermittler, Risikokapitalgeber, Geldtransferdienstleister (PayPal, VISA etc.)

- Marktplätze: Börsen, Auktionshäuser, Online-Plattformen (Amazon, eBay, Uber, AirBnB etc.) 


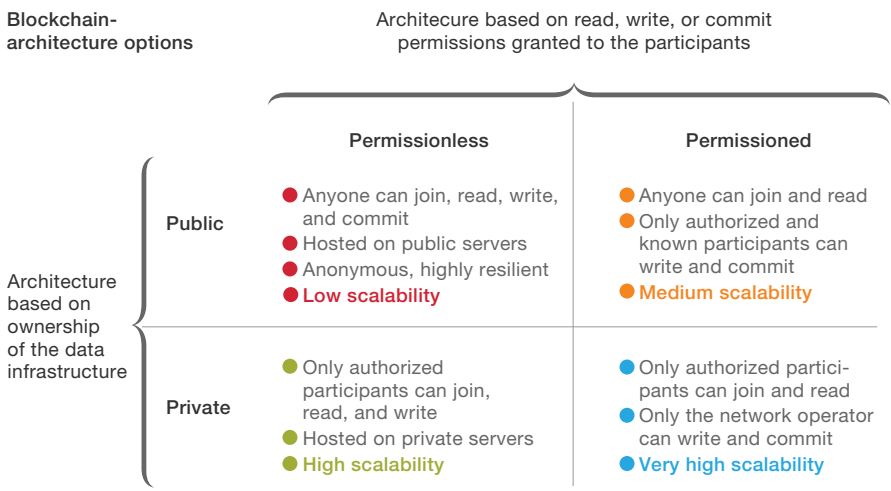

Abbildung 5. Gestaltungsparameter einer Blockchain-Architektur (Quelle: McKinsey [10])

- Hoheitliche Aufgaben: Grundbuchämter, Handelsregister, Notare, Wahlsysteme

- Prüfungs- und Reportingfunktionen: Accounting, Audit, Compliance, Controlling, Wirtschaftsprüfer

Im Grunde waren Trusted Third Parties bislang unerlässlich, da ansonsten nie verlässlich werthaltige Informationen über digitale Transaktionen ausgetauscht werden könnten. Am Einfachsten kann man sich dies am Beispiel eines Börsenbetreibers veranschaulichen: Ohne den Betreiber, der potentielle Käufer und Verkäufer von Wertpapieren zusammen bringt und für jene nach Abschluss des Geschäfts den bilateralen Tausch Geld gegen Wertpapiere ${ }^{13}$ durchführt, konnte bislang kein digitaler Wertpapierhandel betrieben werden. Sie erbrachten also eine überaus wichtige Funktion, ohne die solche digitalen Transaktionen nicht möglich gewesen wären.

Es gibt jedoch einige Nachteile von Trusted Third Parties, die durch die Blockchain-Technologie größtenteils behoben werden: Im Allgemeinen verlangen Trusted Third Parties für die Erbringung ihrer Dienstleistung - nämlich Herstellung von Vertrauen in digitalen Transaktionen - eine Gebühr. Der Abschluss des Transaktionsprozesses wird zu- dem meist verlangsamt, da eine weitere Instanz involviert ist. ${ }^{14}$ Und zuletzt erhöht sich durch das Zwischenschalten einer dritten Partei das Risiko, böswilligen Manipulationen, Sicherheitsrisiken oder Fehlern ausgesetzt zu sein.

Die Blockchain-Technologie verheißt eine Verbesserung dieser Situation: Die Transaktionskosten können reduziert, wenn auch nicht vollständig eliminiert, werden und, da keine dritte Partei involviert ist, können gleichzeitig die Prozesse nach Abschluss der Transaktion effizienter in der verteilten Datenbank durchgeführt werden - oft sogar durch Algorithmen, die eine autarke Ausführung von Anweisungen in dezentraler Weise ermöglichen. ${ }^{15}$ Die Abhängigkeit von Intermediären wird natürlich durch die Nutzung einer verteilten Datenbank und digitaler Signaturen eliminiert. Der Ablauf einer typischen Transaktion über ein BlockchainNetzwerk ist in Abbildung 6 dargestellt.

Zusammengefasst lassen sich die Potentiale der Blockchain-Technologie in vier verschiedene Aspekte einteilen, wobei meist in konkreten Anwendungen hiervon mehrere gleichzeitig verwendet werden:

1. Verteilte Speicherung: In einer Blockchain lassen sich Einträge - und damit auch Werte, wie beispielsweise Besitz-
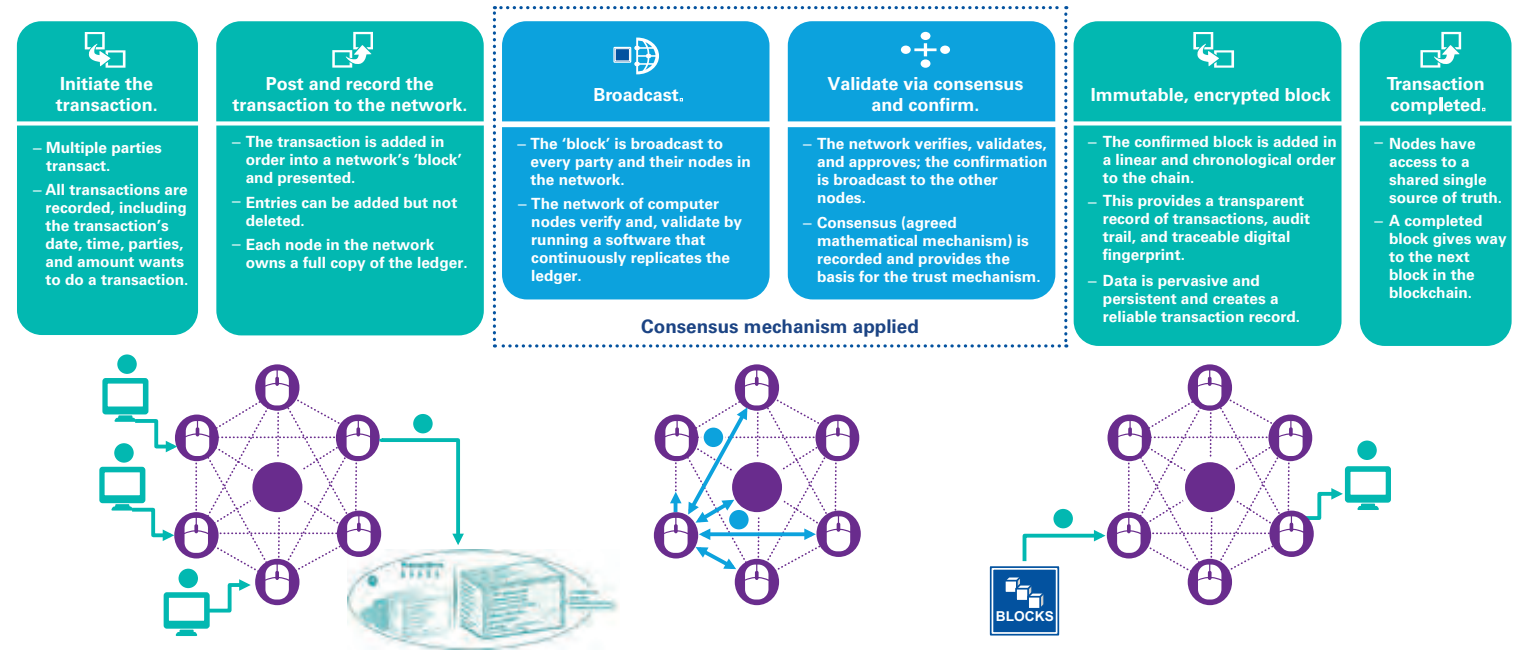

Abbildung 6. Ablauf einer Transaktion in einem Blockchain-Netzwerk (Quelle: KPMG [8]) 
rechte - kryptografisch abgesichert in dezentraler Weise speichern.

2. Konsens-Bildung: Die Validität der dezentral gespeicherten Einträge wird durch einen festgelegten Algorithmus geregelt, weshalb die bisherigen Intermediäre überflüssig werden.

3. Nachvollziehbarkeit und Transparenz: Da die Transaktionen digital signiert und mit einem Zeitstempel versehen, unveränderlich abgelegt werden, lassen sich diese auch für weitere (berechtigte) Beteiligte sowie im zeitlichen Verlauf bei Änderungen, transparent nachvollziehen.

4. Automatisierung: Über eine Automatisierung auf Basis der Blockchain lassen sich Prozesse vertrauenswürdig algorithmisieren. ${ }^{16}$

Zugegebenermaßen lassen sich viele der Potentiale auch auf herkömmliche Weise, in einem zentralen Ansatz, erreichen. Jedoch ist hierbei meist im Vorfeld eine Einigung auf ein konkretes Zielbild sowie entsprechende Rollenverteilungen und Verantwortlichkeiten der Teilnehmer erforderlich, was durch die nicht immer abgestimmten Zielsetzungen und das potentielle Misstrauen der involvierten Parteien untereinander erschwert wird. Andernfalls wäre nur schwer zu erklären, weshalb diese Vorteile nicht schon längst in der Praxis realisiert worden sind.

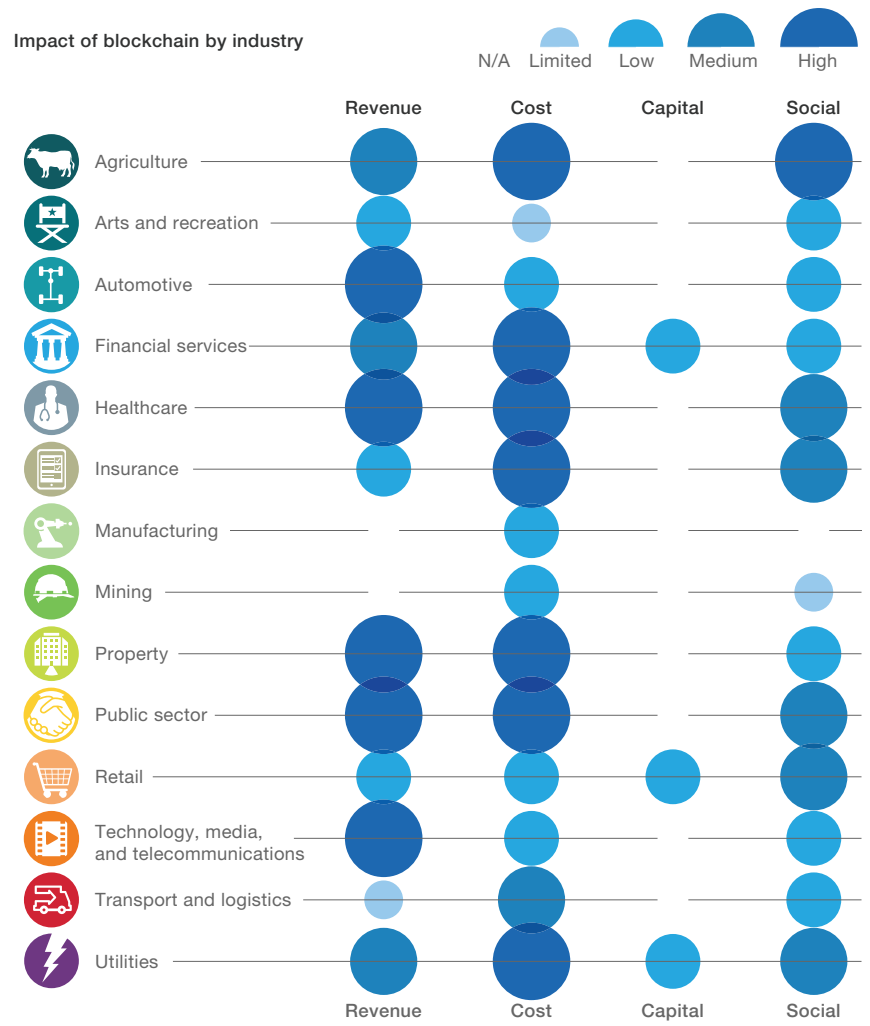

Abbildung 7. Blockchain-Auswirkungen nach Branchen (Quelle: McKinsey [10])
Aus Sicht der Geschäftswelt gibt es vielfältige Anwendungsmöglichkeiten der Blockchain. In Abbildung 7 werden die Auswirkungen der Blockchain-Technologie in den verschiedenen Branchen zusammenfassend dargestellt. Als grobe Strukturierung der unterschiedlichen Anwendungen hilft oft eine Gliederung der Anwendungen in drei Ebenen: Die Ebene eines einzelnen Unternehmens, die brancheninterne und die branchenübergreifende Ebene.

\subsection{Unternehmensebene}

Auf der Ebene eines Unternehmens können insbesondere durch das Ausschalten von Intermediären Kosten- und Effizienzvorteile realisiert werden, da Intermediäre für ihre Dienstleistungen eine Provision verlangen. Beispielsweise könnten Versicherungsmakler oder Bankberater in ihrer Rolle als Intermediäre durch Peer-to-Peer-Transaktionen überflüssig und die Provision deutlich verringert werden. Dies führt branchenübergreifend für die Intermediäre in solchen Transaktionen dazu, dass sie den Mehrwert, den sie zu einer derartigen Transaktion beisteuern und wofür sie entlohnt werden wollen, klarer herausstellen müssen. Dies war in der Vergangenheit nicht immer der Fall und deshalb ist diese Entwicklung gesamtgesellschaftlich, aber auch ökonomisch sicherlich zu begrüßen. ${ }^{17}$

Des Weiteren könnten in multinationalen Organisationen bislang umständliche Abstimmungsprozesse, wie beispielsweise Konsolidierungen von Einzelbilanzen in Konzernen oder auch unternehmensinterne Geldtransaktionen über Landesgrenzen hinweg, vereinfacht werden, da es nur eine einzige Datengrundlage (Wahrheit) gibt, die bei den unterschiedlichen Beteiligten verteilt gespeichert ist. Eine gleichzeitige Automatisierung auf dieser Basis führt zu stark veränderten Geschäftsmodellen, in welchen viele der zugehörigen Prozesse oder erzeugten Produkte ohne manuelle Interaktion durchgeführt werden können (Algorithmisierung des Business). Ein konkretes, wenn auch zugegebenermaßen sehr einfaches Beispiel für ein solches Produkt auf Basis der Blockchain ist die Versicherung gegen Flugverspätungen der AXA. ${ }^{18}$ Diese zahlt vollkommen autark bei einer Verspätung des versicherten Flugs die vereinbarte Entschädigungssumme an den Versicherungsnehmer aus.

Durch die Blockchain wird auch eine Tokenisierung von physischen und (neben Zahlungsmitteln) weiteren virtuellen Gütern - wie Patenten, Zeugnissen, Gutscheinen, Verbindlichkeiten, digitaler Kunst oder auch in-game items auf einfache Weise ermöglicht. Dabei wird ein virtuelles Abbild ${ }^{19}$ der einzelnen Güter erstellt und mit nichtduplizierbaren Token versehen, die dann auf Plattformen gehandelt werden können oder hierüber für manche Beteiligte, wie Privatpersonen, überhaupt erst handelbar oder verlässlich geteilt werden können. Kryptowährungen, und insbesondere Bitcoin, waren hierfür nur das erste und sicherlich einfachste Beispiel. Fortgeschrittene Realisierungen einer Tokenisierung von physischen Gütern sind beispielsweise heute schon für Diamanten ${ }^{20}$, Energie $^{21}$, Immobilien $^{22}$ oder auch Fahrzeuge ${ }^{23}$ vorhanden. 


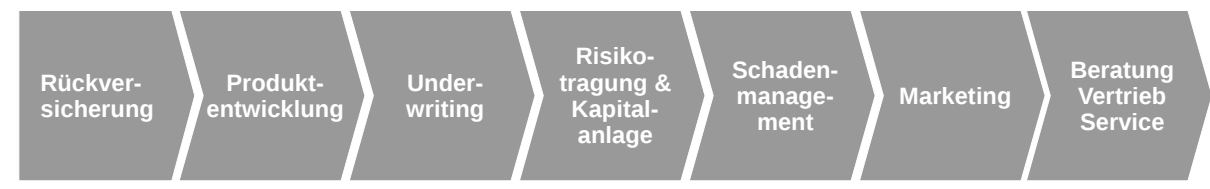

Abbildung 8. Vereinfachte Wertschöpfungskette einer Versicherung

Im Extremfall ist es sogar vorstellbar, dass die Blockchain zu einer Auflösung der existierenden Wertschöpfungsketten führt. Eine Wertschöpfungskette repräsentiert in einer einfachen, schematischen Form unter anderem die unterschiedlichen Informationsflüsse innerhalb eines $\mathrm{Ge}$ schäftsmodells (in Abbildung 8 ist exemplarisch die Wertschöpfungskette eines Versicherungsunternehmens dargestellt).

Der Zweck eines Unternehmens wird durch die effiziente Verbindung der einzelnen Teile der Wertschöpfungskette begründet. Wenn auch in der Vergangenheit die Bereitstellung von Teilen der eigenen Wertschöpfungskette an externe Firmen (Outsourcing) abgegeben wurde, blieben die individuellen Kernelemente stets in der Hand des Unternehmens, da sie den Wettbewerbsvorteil - wenn nicht sogar die Existenzberechtigung - darstellten.

Speziell das Geschäftsmodell eines Versicherers wird seit einigen Jahren durch unterschiedliche Entwicklungen herausgefordert. In nachstehenden vier Bereichen sind signifikante Veränderungen spürbar:

(a) Kapitalanforderungen: Im Gegensatz zu früher hindern Liquiditäts- und Solvenzanforderungen neue Wettbewerber nicht mehr daran, in die Versicherungsbranche einzusteigen, da über alternative Finanzierungsmöglichkeiten, wie Crowdfunding ${ }^{24}$, Kapital zur Verfügung gestellt werden kann.

(b) Kosten: Der Kostendruck innerhalb der Versicherungsbranche steigt weiter, da Transaktionskosten durch die Automatisierung von Aufgaben, beispielsweise mit Hilfe von künstlicher Intelligenz, massiv unter Druck geraten.

(c) Standardisierung und Transparenz: Versicherungsprodukte und -dienstleistungen werden mehr und mehr über modulare Komponenten standardisiert und verwenden granularere Datengrundlagen. Folglich sind sie in sehr viel stärkerem Maße als früher zwischen konkurrierenden Versicherungsunternehmen vergleichbar sowie global skalierbar. Letzteres wird insbesondere bei kleineren und mittelgroßen Versicherungsgesellschaften zusätzlichen Druck auf die Kostensituation ausüben (dieser Wandel ist dem der Automobilbranche sehr ähnlich, in welchem ein modularer Plattformansatz heutzutage allgegenwärtig 25 ist und nur noch eine geringe Anzahl von lokal-aufgestellten Herstellern existieren).

(d) Nähe zum Kunden: Über digitale Wege können Anbieter ihre Dienstleistungen viel einfacher als früher, direkt an Kunden und Geschäftspartner vertreiben. Darüber hinaus wird dieser Wettbewerb über neue Arten von
Versicherungsprodukten - exemplarisch seien Peer-toPeer-, Mikro- oder auch Kurzzeitabsicherungen genannt - noch weiter verschärft.

Ein Versicherungsunternehmen ist nichts anderes als ein Intermediär - oder Marktplatz - in einer Transaktion zwischen zwei Beteiligten. Einer dieser Beteiligten möchte ein spezifisches Risiko absichern; der andere ist bereit, dieses Risiko zu tragen und verlangt hierfür im Gegenzug eine Bezahlung (Versicherungsprämie). In der Vergangenheit war dieser Marktplatz weder transparent noch allzu liquide, da vor allem die von der Finanzdienstleistungsaufsicht (BaFin) vorgegebenen Kapitalanforderungen sowie die fehlende Datengrundlage und Expertise, wie zum Beispiel über historische Schadenverläufe, hinderlich waren. Ähnlich wie bei den Banken werden jedoch nun auch Versicherungsunternehmen, insbesondere durch das Aufkommen von Startups aus dem FinTech- und InsurTech-Bereich ${ }^{26}$, welche in intelligenter Weise Technologie auf die Finanzdienstleistungsbranche anwenden, in den Kernelementen ihrer Wertschöpfungskette bedroht. ${ }^{27}$

Die Blockchain könnte gerade für die Finanzdienstleistungsbranche, die keine physischen Güter produziert, sondern nur mit Daten sowie zukunftsgerichteten Modellen arbeitet, eine technologische Infrastruktur bieten, in welchem die einzelnen Teile der Wertschöpfungskette durch eigenständig operierende Unternehmen (oder Algorithmen) mit einer speziellen Expertise auf ihrem Gebiet erbracht werden. Der Informationsfluss zwischen den Teilen wird über Transaktionen, die dann dezentral bei den verschiedenen Beteiligten gespeichert sind, vollzogen. Kryptografisch abgesichert, kann dies in der Theorie den bislang vorhandenen Abstimmungsbedarf zwischen den einzelnen Teilen automatisieren, wenn nicht gar vollständig eliminieren. Die Geschäftstätigkeit eines Unternehmens könnte sich dadurch stark verändern. Es würde höchstens noch ein Beteiligter von Vielen in der Erbringung der Dienstleistung sein. Das könnte in der Versicherungsbranche zu einem dezentralen Marktplatz für Versicherungen führen.

Solche Ansätze (Decentralizing Insurance) basierend auf Blockchain sind keine abstrakten Gedankenspiele, sondern existieren bereits in der Realität und sollten deshalb, trotz ihres jungen Alters, keinesfalls unterschätzt werden. Ein durchdachtes Beispiel hierfür ist das Startup Ethe$\operatorname{risc}^{28}$, das sich zum Ziel gesetzt hat, Versicherungsunternehmen wie sie heute existieren, überflüssig zu machen. Für weiterführende Gedanken zur Anwendung von Blockchain im Versicherungsbereich, siehe auch die Beiträge [7] oder [6]. 


\subsection{Brancheninterne Ebene}

Auch innerhalb von Branchen gibt es viele Informationsflüsse zwischen den einzelnen Unternehmen oder deren Produkten, wobei natürlich nicht potentiell widerrechtliche Absprachen gemeint sein sollen.

In der Automobilbranche müssen beim Platooning, bei dem mehrere Fahrzeuge in sehr engem Abstand hintereinander fahren können, Informationen über Position und Geschwindigkeit zwischen den Fahrzeugen unterschiedlicher Hersteller ausgetauscht werden; ein sehr ähnlicher Fall ist das Zukunftsszenario autonomer Fahrzeuge, die untereinander, aber auch mit anderen Verkehrsteilnehmern und Anlagen kommunizieren müssen - hierfür ist eine vertrauenswürdige Infrastruktur notwendig.

Ein zweites Beispiel ist die Energiebranche: Wenn Energie lokal erzeugt und auf Peer-to-Peer-Basis verkauft werden soll (vgl. auch [13]), müssen die zu Grunde liegenden technischen Komponenten natürlich kompatibel sein; neben der einfacheren Handelbarkeit, die möglicherweise über Tokens realisiert würde, kann eine Blockchain-Infrastruktur in der Energiebranche auch für Netzoptimierungszwecke zwischen den verschiedenen Energieerzeugern verwendet werden. Die Informationsflüsse zwischen den beteiligten Unternehmen sind leicht erkennbar und entsprechende Schnittstellen bzw. eine Plattform, möglichst ohne zentralen Intermediär, schaffen das größtmögliche Vertrauen.

Zuletzt noch ein detaillierter Blick auf die Versicherungsbranche, in welcher sehr viele Informationsflüsse zwischen Wettbewerbern existieren. Hierzu zählen beispielsweise die Folgenden:

- Schadenfreiheitsklasse: In der Kraftfahrzeugversicherung hat sich das System der Schadenfreiheitsklassen etabliert. ${ }^{29}$ Damit dieses auch unternehmensübergreifend funktionieren kann und den Kunden der Wechsel nicht erschwert bzw. unmöglich gemacht wird, müssen konkurrierende Versicherungsgesellschaften die spezifischen Informationen bei einem Wechsel der Versicherungsgesellschaft durch den Kunden übermitteln.

- Mehrfachversicherung: In den meisten Versicherungsverträgen ist die Mehrfachversicherung ${ }^{30}$ einer Gefahr ausgeschlossen. Um ihre jeweiligen Zahlungsverpflichtungen im Schadensfall bestimmen zu können, müssen Versicherungsgesellschaften Informationen untereinander austauschen.

- Betrugsvermeidung: Das Hinweis- und Informationssystem (HIS) der deutschen Versicherungswirtschaft ist ein zentrales System, das Versicherungsbetrug bekämpfen und die Risikoprüfung effizient gestalten will. Es soll die jeweiligen Versichertengemeinschaften vor „fehlerhaften, unwahren, unvollständigen oder betrügerischen Angaben schützen“, wodurch höhere Beiträge für die Versicherungsnehmer vermieden werden. Das HIS hilft Versicherern bei der Antragsprüfung die Risikobewertung präziser vorzunehmen. Hierfür ist der Informationsaustausch innerhalb der Branche unerlässlich.

- Konsortialversicherungen: Dies sind Versicherungsgeschäfte von Versicherungen, die diese nicht alleine, sondern im Rahmen eines Konsortiums für ihre Versicherungskunden durchführen. Dass hierfür zwischen den beteiligten
Konsortialpartnern Informationen geteilt werden müssen, steht außer Frage.

- Rückversicherung: Für die Inanspruchnahme von Rückversicherung müssen Versicherer Informationen über ihr Versicherungsportfolio mit der Rückversicherungsgesellschaft teilen.

Auf Grund der letztgenannten Beispiele und vieler weiterer hat der Autor des vorliegenden Artikels im Jahr 2016 den Anstoß zur Blockchain Insurance Industry Initiative (B3i) (http://b3i.tech) gegeben. In dieser haben seitdem konkurrierende Versicherungsunternehmen in einem offenen Dialog über die Möglichkeiten zur Anwendung der BlockchainTechnologie in der Versicherungsbranche diskutiert. Diese Gespräche waren überaus erfolgreich und führten im März 2018 zur Gründung der B3i Services AG als Joint Venture, an welchem sich 13 globale Versicherungsgesellschaften ${ }^{31}$ als Gründungsaktionäre beteiligt haben:

B3i Services AG is a startup formed to explore the potential of using Distributed Ledger Technologies within the re/insurance industry for the benefit of all stakeholders in the value chain. B3i provides insurance solutions on a blockchain platform offering opportunities for efficiency, growth and quality across the value chain to benefit all participants including end customers.

Das Joint Venture hat sich zum Ziel gesetzt, die Transaktionen zwischen den einzelnen Versicherungsunternehmen effizienter auf Blockchain-Basis durchzuführen und damit auch Kosten einzusparen, was über eine entsprechende Standardisierung und Automatisierung von Prozessen gelingen könnte. Die langfristige Vision von B3i ist es, Versicherungsprodukte für Verbraucher relevanter, leichter zugänglich und erschwinglicher zu machen und gleichzeitig ein Ökosystem bereit zu stellen, das innovative Lösungen und Produkte in der Versicherungsbranche ermöglicht. Mit Blockchain wird die Qualität der Datenbereitstellung erhöht und perspektivisch sind völlig neue Geschäftsmodelle denkbar, die ohne Blockchain nur schwer realisierbar wären. Erste Anwendungsfälle sowie weitere vielversprechende Prototypen sind bereits in der Umsetzung.

In einem vergleichbaren Ansatz wie $B_{3} i$ hat auch der US-amerikanische Versicherungsverband ein Versicherungskonsortium mit dem Namen RiskBlock ${ }^{32}$ gegründet und ähnliche Gründungen von Konsortien wurden mittlerweile auch in den anderen, oben aufgeführten Branchen voran getrieben:

- Automobilbranche: In der globalen Mobility Open Blockchain Initiative (Mobi) 33 haben sich unter anderem die Automobilhersteller BMW, Ford, GM und Renault sowie die Automobilzulieferer Bosch und ZF beteiligt: "Mobi is a nonprofit organization working with forward thinking companies, governments, and NGOs to make mobility services more efficient, affordable, greener, safer, and less congested by promoting standards and accelerating adoption of blockchain, distributed ledger, and related technologies." 
- Energiebranche: In der deutschen Energiebranche ist EnerChain ${ }^{34}$ das vorherrschende Blockchain-Konsortium, in welchem sich unter anderem EnBW, Eon, RWE, Vattenfall und Uniper einbringen: "Enerchain is a Blockchain based distributed ledger that has the potential to become the operating system of Energy Trading covering the entire trade cycle from end to end."

Der Autor ist der festen Überzeugung, dass sich BlockchainPlattformen in vielen, wenn nicht sogar allen Branchen als Standard zum Austausch von Information und der dauerhaften dezentralen Abspeicherung von Daten durchsetzen werden. Die beschriebenen Beispiele deuten jedenfalls stark in diese Richtung und viele weitere Konsortialansätze - Logistik, Handelsfinanzierung, Luftfahrt - könnten zusätzlich angeführt werden.

\subsection{Branchenübergreifende Ebene}

In der branchenübergreifenden Ebene steht oft die einzelne Person bzw. dessen digitale Identität im Zentrum des Geschehens und es müssen Informationen aus den unterschiedlichen Branchen geteilt werden. Als konkretes Beispiel sei die Zulassung eines Fahrzeugs bei der Zulassungsstelle genannt. Hier werden unterschiedliche Datenpunkte aus verschiedenen Branchen benötigt - insbesondere der KFZ-Brief (vom Automobilhersteller), eine Versicherungsbestätigung (vom Versicherungsunternehmen) und ein Ausweisdokument (vom Staat). Um den Prozess der Fahrzeuganmeldung aus Bürgersicht digital einfach zu gestalten, könnte die Blockchain mitunter als Katalysator eingesetzt werden. Bestehende Überlegungen von Unternehmen, staatlich geförderten Einrichtungen sowie Hochschulen gehen bereits in diese Richtung, was der Autor begrüßt. Das schwierige Feld des E-Government 35 bietet, wie viele Leser vermutlich aus persönlicher Erfahrung wissen, noch reichlich Verbesserungspotential ${ }^{36}$ und Blockchain kann hier eine Technologie sein, die Fortschritte ermöglicht. Denkbar ist auch, dass sich ein System von Meta-Blockchains etabliert, in welchem die einzelnen Blockchain-Implementierungen von Branchen- und Unternehmen miteinander verknüpft sind. Diese Meta-Blockchains wären dann für den Austausch der branchenunabhängigen Informationen, wie beispielsweise Geldflüssen oder auch digitalen Identitäten bzw. Identitätsmerkmalen, zuständig. Hierfür ist im Besonderen der Staat als Impulsgeber und Akteur weiter gefragt.

\section{Ausblick}

Zusammenfassend unternahm dieser Artikel den Versuch, die Relevanz von Blockchain aus der Business-Perspektive durch konkrete Beispiele zu veranschaulichen und somit ein äußerst spannendes Feld in der breiteren Öffentlichkeit für praktische Anwendungen der Mathematik und Informatik aufzuzeigen. Was die Zukunft mit Blockchain bringen wird, ist leider wie immer schwer zu prognostizieren. Dass die Blockchain-Technologie weiterhin bestehen und angewandt werden wird, steht jedoch außer Frage - wenn auch einige der aktuellen Diskussionen zu möglichen Einsatzfeldern dem Autor etwas unrealistisch erscheinen.
Die beiden Komponenten der Blockchain, Kryptografie und verteilte Netzwerke, bieten jedoch auch einzeln noch sehr viel unausgeschöpftes Anwendungspotential,37 beispielsweise wenn diese vor dem Hintergrund des Datenschutzes oder der digitalen Souveränität betrachtet werden. Große Unternehmen - hier sei das Unternehmen Apple als ein prominentes Beispiel angeführt - nutzen zum Teil bereits kryptografische Verfahren ${ }^{38}$, um den rechtlichen, aber vor allem auch den aus moralischen Gründen auferlegten Erfordernissen genügen zu können. In den nächsten Jahren werden daher diese theoretischen Grundlagen aus der Mathematik noch weiter an Relevanz gewinnen und die Blockchain hat trotz mancher negativer Schlagzeilen über sie mit ihrem großen Bekanntheitsgrad diesbezüglich bereits für erste Denkanstöße in vielen Unternehmen und auch der Gesellschaft gesorgt, was durchaus zu begrüßen ist.

\section{Anmerkungen}

1. Siehe beispielsweise $[1,2,10,15,16]$.

2. Vgl. Introducing a16z crypto (https://a16zcrypto.com/)

3. Gartner's Hype Cycle for Emerging Technologies, 2017

4. Mit dem Double-Spending-Problem wird eine potentielle Schwachstelle digitaler Währungen bezeichnet, bei der derselbe Token (digitale Repräsentanz der Geldeinheit) mehrfach eingesetzt wird. Dies ist vorstellbar, da Informationen, die digital vorliegen, relativ einfach dupliziert werden können. Ein erfolgreiches Double-Spending führt neben der Inflation des Geldwerts zu einem Vertrauensverlust in das System. Präventionsmechanismen zur Verhinderung des Double-Spendings hatten bis zur Einführung von Bitcoin einen zentralen Charakter. Das bekannteste Beispiel ist der Internet-Zahlungsdienstleister PayPal, welcher in einer zentralen Datenbank alle Geldtransaktionen registriert. 5. In den meisten Fällen befinden sich mehrere Transaktionen gebündelt in einem Block.

6. Im Englischen als Distributed Ledger bezeichnet.

7. Der Grad an Transparenz lässt sich jedoch adjustieren und an den jeweiligen Bedarf des konkreten Anwendungsfalls anpassen.

8. Oft werden die Bezeichnungen dezentral und verteilt auch synonym verwendet.

9. IOTA: The Next Generation of Distributed Ledger Technology (http://www.iota.org)

10. R3: Blockchain for business (https://www.r3.com)

11. Dies wird auch als byzantinischer Fehler bezeichnet: Als solchen bezeichnet man in der Informationstechnik Fehler, bei denen sich ein System beliebig falsch verhalten kann.

12. Der Energieverbrauch entsteht durch das Bitcoin-Mining.

13. Clearing und Settlement

14. Dies lässt sich wieder am Beispiel eines Börsenbetreibers gut illustrieren: Die Prozesse nach einer Transaktion dauern mindestens einige Stunden, bis hin zu mehreren Tagen. Erst nach erfolgreichem Abschluss hat das Wertpapier faktisch den Besitzer gewechselt und der neue Eigentümer kann über dieses verfügen. Diese Ineffizienz ist jedoch kein technisches Problem.

15. Dies wird auch mit dem Begriff Smart Contracts bezeichnet, auch wenn es sich dabei offensichtlich nicht um Verträge im juristischen Sinne handelt.

16. Ein extremes Beispiel hierfür ist die Gründung der Decentralized Autonomous Organization (DAO) im Jahr 2016, die mehr als \$140 Millionen eingesammelt hat, vgl. den Artikel „Die erste Firma ohne Menschen“ aus der ZEIT [5].

17. Auch wenn es für einzelne Intermediäre sicherlich zu einer Veränderung ihrer bisherigen Arbeitsweise führen mag.

18. AXA's fizzy: Smart insurance. Automatic compensation. (https://fizzy. axa/)

19. Oft auch Digital Twin

20. Everledger: Everledger is a global startup that uses the best of emerging technology including blockchain, smart contracts and machine vision 
to assist in the reduction of risk and fraud for banks, insurers and open marketplaces. (https://www.everledger.io/)

21. SIEMENS: „Siemens und US-Start-up $\mathrm{LO}_{3}$ Energy kooperieren bei Blockchain-Microgrids." [13], [14]

22. Harbor: "Real estate assets [...] can benefit greatly from the improved efficiency, transparency, liquidity, and fractional ownership possibilities of blockchains." (https://harbor.com/)

23. AXA Winterthur: „Car Dossier ist eine digitale Plattform auf Blockchain-Basis, auf der alle relevanten Informationen über den gesamten Lebenszyklus eines Autos gesammelt werden."

24. Dies wird auf Blockchain-Basis oft über ein Initial Coin Offering (ICO), einer Emission von Tokens, dargestellt.

25. Dies birgt allerdings auch Nachteile, was das Beispiel des aktuellen Dieselskandals in Zusammenhang mit der Audi-Motorenfertigung vor Augen führt.

26. Financial Technology \& Insurance Technology

27. Dies wird im Englischen oft mit dem Begriff Unbundling of the value chain bezeichnet.

28. Etherisc: "Decentralized insurance protocol to collectively build insurance products" (https://etherisc.com)

29. Das System der Schadenfreiheitsklassen ermöglicht eine präzisere Risikoeinschätzung eines Fahrers basierend auf seinem historischen Unfallverlauf. Konkret wird ein Rabatt auf die Versicherungsprämie gewährt, dessen Höhe sich nach der Anzahl der unfallfreien Jahre bemisst.

30. „Ist bei mehreren Versicherern ein Interesse gegen dieselbe Gefahr versichert [...] haften die Versicherer in der Weise als Gesamtschuldner [... ] der Versicherungsnehmer aber insgesamt nicht mehr als den Betrag des Schadens verlangen kann." ( $\$ 78$ VVG)

31. Unter anderem Allianz, Generali, Hannover Re, Munich Re, Scor, Swiss Re und Zurich.

32. "Industry leaders are joining forces to unlock the potential of blockchain." (https://www.theinstitutes.org/guide/riskblock)

33. https://www.dlt.mobi

34. https://enerchain.ponton.de

35. Das schweizerische Startup Procivis ist hier sicherlich als Vorreiter zu nennen, denn es will e-Government as a Service anbieten. (https://procivis.ch/)

36. Am Rande sei angemerkt, dass das Wort Blockchain mehrfach im Koalitionsvertrag der aktuellen Bundesregierung als zukünftiger Forschungsschwerpunkt genannt wird.

37. Dies wird als CryptoTech und Distributed Ledger Technologies (DLT) bezeichnet.

38. Privacy-Enhancing Technologies, wie unter anderem Differential Privacy oder Zero-Knowledge-Proofs

\section{Literatur}

[1] The trust machine. The Economist, Oktober 2015.

[2] The blockchain issue: The future of trust? MIT Technology Review, Mai 2018.

[3] Paul Baran. On distributed communication networks. IEEE Transactions of the Professional Technical Group on Communication Systems, CS-12(1), März 1964.

[4] Miguel Castro and Barbara Liskov. Practical Byzantine fault tolerance. Proceedings of the Third Symposium on Operating Systems Design and Implementation, 1999.

[5] Die ZEIT. Die erste Firma ohne Menschen. tinyurl.com/ y9zqhpqz, Mai 2016.

[6] Markus Hablizel, Stephan Janssens, and Sebastian Steger. Blockchain can bring insurance back to the basics. tinyurl.com/ y9vjy6jm, Oktober 2016.

[7] Patrick Hagen. Blockchain-Technologie verunsichert die Assekuranz. Herbert Frommes Versicherungsmonitor, 24. November 2016.

[8] KPMG. Consensus: Immutable agreement for the internet of value, Juni 2016

[9] Leslie Lamport, Robert Shostak, and Marshall Pease. The Byzantine generals problem. ACM Transactions on Programming Languages and Systems, 4(3):387-389, 1982.

[10] McKinsey \& Company. Blockchain beyond the hype: What is the strategic business value? Juni 2018.

[11] Satoshi Nakamoto. Bitcoin: A peer-to-peer electronic cash system, November 2008. https://bitcoin.org/bitcoin.pdf.

[12] Arvind Narayanan, Joseph Bonneau, Edward Felten, Andrew Miller, and Steven Goldfeder. Bitcoin and Cryptocurrency Technologies: A Comprehensive Introduction. Princeton University Press, 2016.

[13] SIEMENS. Siemens und US-Start-up $\mathrm{LO}_{3}$ Energy kooperieren bei Blockchain-Microgrids tinyurl.com/ydanl8kc, November 2016.

[14] SIEMENS. Siemens investiert in $\mathrm{LO}_{3}$ Energy und stärkt bestehende Partnerschaft. tinyurl.com/y933jctw, Dezember 2017.

[15] Mark Walport. Distributed ledger technology: Beyond block chain. United Kingdom's Government Office for Science, Januar 2016.

[16] World Economic Forum. The future of financial infrastructure: An ambitious look at how blockchain can reshape financial services, August 2016. 